\title{
Co-culturing human prostate carcinoma cells with hepatocytes leads to increased expression of E-cadherin
}

\author{
CC Yates', CR Shepard', DB Stolz ${ }^{2}$ and A Wells*,I \\ 'Department of Pathology, University of Pittsburgh, and Pittsburgh VAMC, Pittsburgh, PA 152 I3, USA; ${ }^{2}$ Department of Cell Biology, University of \\ Pittsburgh, and Pittsburgh VAMC, Pittsburgh, PA 15213, USA
}

Metastasis is a multi-step process wherein tumour cells detach from the primary mass, migrate through barrier matrices, gain access to conduits to disseminate, and subsequently survive and proliferate in an ectopic site. During the initial invasion stage, prostate carcinoma cells undergo epithelial-mesenchymal-like transition with gain of autocrine signalling and loss of E-cadherin, hallmarks that appear to enable invasion and dissemination. However, some metastases express E-cadherin, and we found close connections between prostate carcinoma cells and hepatocytes in a liver microtissue bioreactor. We hypothesise that phenotypic plasticity occurs late in prostate cancer progression at the site of ectopic seeding. Immunofluorescence staining for E-cadherin in co-cultures of hepatocytes and DU- 145 prostate cancer cells revealed E-cadherin upregulation at peripheral sites of contact by day 2 of co-culture; E-cadherin expression also increased in PC-3 cells in co-culture. These carcinoma cells bound to hepatocytes in an E-cadherindependent manner. Although the signals by which the hepatocytes elicited E-cadherin expression remain undetermined, it appeared related to downregulation of epidermal growth factor receptor (EGFR) signalling. Inhibition of autocrine EGFR signalling increased E-cadherin expression and cell-cell heterotypic adhesion; further, expression of a downregulation-resistant EGFR variant prevented E-cadherin upregulation. These findings were supported by finding E-cadherin and catenins but not activated EGFR in human prostate metastases to the liver. We conclude that the term epithelial-mesenchymal transition only summarises the transient downregulation of E-cadherin for invasion with re-expression of E-cadherin being a physiological consequence of metastatic seeding. British Journal of Cancer (2007) 96, 1246- 1252. doi:I0.1038/sj.bjc.6603700 www.bjcancer.com Published online 3 April 2007 (c) 2007 Cancer Research UK

Keywords: EGF receptor; centrifugal assay for cell adhesion; heterotypic cell-cell adhesion; epithelial-mesenchymal transition

Metastasis causes the vast majority of the morbidity and mortality from cancer. However, our understanding of the multi-step cascade of events that must occur for successful attachment and subsequent metastasis has not been completely elucidated, as few systems are available to examine the intermediary dynamic steps that occur during progression (Condeelis and Segall, 2003; Yates et al, 2007). Only now are investigators defining the changes needed for both a cell to escape from the primary tumour and subsequently to allow for ectopic survival and proliferation.

An essential step for cells to migrate from the primary tumour mass is the loss of epithelial cell-cell adhesions and subsequent acquisition of a mesenchymal-like migratory and invasive phenotype, generally referred to as epithelial-mesenchymal transition (EMT) (Thiery, 2002; Ackland et al, 2003; Bates and Mercurio, 2005). Central to this dissociation from the primary tumour is altered or loss of E-cadherin expression (Davies et al, 2000; Lowy et al, 2002) a key attribute that may define the EMT. Ecadherin is a calcium $\left(\mathrm{Ca}^{2+}\right)$-dependent transmembrane cell surface glycoprotein. E-cadherin rapidly localises on the cell surface to regions of contact, usually resulting in homotypic cell-

*Correspondence: Dr A Wells; E-mail: wellsa@upmc.edu Received 29 August 2006; revised 22 February 2007; accepted 22 February 2007; published online 3 April 2007 cell binding; this fosters the maintenance of normal cellular structure. Recently, reports have shown that aberrant loss or downregulation of E-cadherin in carcinomas may result from reversible epigenetic events (Graff et al, 1995; Lind et al, 2004) or growth factor-mediated downregulation (Hurtubise and Momparler, 2004; Wheeler, 2005), suggesting that loss of this tumour suppressor may be reversible. That this reversal might happen has been suggested by the findings that secondary tumour metastases express E-cadherin (Brabletz et al, 2001; Rubin et al, 2001). However, that this expression at the distal site represents reexpression of E-cadherin has not been demonstrated.

Activation of the epidermal growth factor receptor (EGFR) downregulates E-cadherin expression (Jawhari et al, 1999; Ackland et al, 2003). This suggested that autocrine EGFR signalling present in prostate carcinomas may contribute to E-cadherin repression in these tumours. This was supported by our recent finding that pharmacological abrogation of EGFR signalling in prostate carcinoma cells reverses decreased E-cadherin expression rendering these cells less invasive and more cohesive (Jawhari et al, 1999; Yates et al, 2005). This re-expression of E-cadherin is similarly observed by close contacts of DU-145 prostate carcinoma cells when cultured within the liver microenvironment (Yates et al, 2007). As prostate cancer metastasises to the liver in over half of all patients with metastatic disease (Ewing, 1922; Shah et al, 2004), we sought to explore the correlative expression levels of EGFR and 
E-cadherin in the presence of parenchymal cells of the liver. We show, herein, that hepatocytes elicit E-cadherin expression in prostate carcinoma cells concomitant with downregulation of EGFR signalling.

\section{MATERIALS AND METHODS}

\section{Materials}

The primary antibodies used were mouse monoclonal antibodies selective for human E-cadherin, human cytokeratin 18 and humanspecific controls tubulin (Santa Cruz Biotechnology, Santa Cruz, CA, USA). Primary antibodies to phospho-EGFR (Cell Signaling, Danvers, MA, USA), $\alpha$-catenin, $\beta$-catenin, p120 (BD Biosciences, San Jose, CA, USA) and vimentin (Dako Laboratories, Carpinteria, CA, USA). Polyclonal antibody to EGFR was used for immunohistochemistry (Santa Cruz). Secondary antibodies for immunofluorescence dyes or horseradish peroxidase-conjugated were obtained from (Jackson Laboratories, Bar Harbor, ME, USA). Anti-E-cadherin blocking antibody (SHE78-7) was obtained from Zymed Laboratories (South San Francisco, CA, USA). The EGFR inhibitor PD153035 and primary antibody to human EGFR (Ab-1), were obtained from Calbiochem (San Diego, CA, USA). Other reagents were obtained from Sigma (St Louis, MO, USA).

\section{Cell lines}

The androgen-independent DU-145 and PC-3 human prostate carcinoma cell line were originally derived from a brain and bone metastasis of a human prostate adenocarcinoma, respectively. A transmodulation-resistant EGFR construct was generated by replacing the PKC-target threonine at amino acid 654 with alanine (A654) and transfected into DU-145 cells (Yates et al, 2005). Prostate cancer cells lines were cultured as described previously (Yates et al, 2005). Red fluorescent protein (RFP) was stably transfected with ds-Red (Clontech, Mountain View, CA, USA) vector containing a neomycin resistance gene in DU-145 cells. RFP expressing cells were selected and maintained in DMEM with $10 \%$ FBS and in the presence of $1000 \mathrm{mg} \mathrm{ml}^{-1} \mathrm{G} 418$ until used for experimentation.

\section{Reverse transcription-PCR}

Total RNA was extracted using TRIzol (Invitrogen, Carlsbad, CA, USA). RT reaction was performed using total RNA as a template and an RT for PCR kit (Invitrogen). PCR amplification was carried out with the following primers: human-specific E-cadherin primers, $5^{\prime}$-gacacccgattcaaagtggg- $3^{\prime}$ and $5^{\prime}$-gtctctcttctgtcttctgag- $3^{\prime}$; showed no homology to rat E-cadherin and $30.2 \%$ homology to mouse E-cadherin. GAPDH primer kit $5^{\prime}$-ccacccatggcaaattccatgg ca- $3^{\prime}$ and $5^{\prime}$-tctaacggcaggtcaggtccacc- $3^{\prime}$ (Stratagene, Cedar Creek, TX, USA) recognises both human and rodent sequences.

\section{Hepatocytes}

Primary rat hepatocytes, $>90 \%$ purified, were obtained by collagenase perfusion (Seglen, 1976) of 150-230 g male eGFP (enhanced green fluorescent protein)-transgenic and WT (wildtype) Sprague-Dawley rats, originally generated by Dr Masaru Okabe (Genome Information Research Center, University of Osaka, Osaka, Japan) and were generously provided by Japan SLC, Inc. (Hamamatsu, Japan). Use of rat hepatocytes enables the identification of the human prostate carcinoma cell proteins using human-specific antibodies. The cells were collected and maintained in Hepatocyte Growth Media (HGM) medium before and during co-culture (Yates et al, 2007). These studies were approved by the University of Pittsburgh IACUC committee.

\section{Co-cultures}

Initial co-cultures consisted of 50000 cells cm$~^{-2}$ of freshly isolated hepatocytes and 2000 cells $\mathrm{cm}^{-2}$ prostate cancer cells. Co-cultures were maintained in serum-free HGM (Yates et al, 2007), and plated on tissue culture dishes pre-treated with $10 \%$ collagen (Upstate, Charlottesville, VA, USA).

\section{Centrifugal assay for cell adhesion}

This assay is a modification of the McClay and Giacolmello assays (McClay et al, 1981). Cancer cells were non-enzymatically dissociated and labelled with $5 \mu \mathrm{m}$ Calcein AM (Molecular Probes, Carlsbad, CA, USA). Labelled cancer cells were seeded at a density of 42000 cells well $^{-1}$ in 96-well plates containing a densely confluent hepatocyte monolayer. The plates were centrifuged for $<60$ s at $50 \mathrm{~g}$ to pellet the cancer cells onto the hepatic monolayer, then incubated at $37^{\circ} \mathrm{C}$. At defined times, the plates were inverted and centrifuged at $600 \mathrm{~g}$ for $5 \mathrm{~min}$ and then gently washed to remove unbound cells from the hepatocyte monolayer. Fluorescence was measured with a 494/517 bandpass filter set-up from the bottom of the plate by a TECAN Spectra-Fluor plate fluorometer. Absolute emission measurements were background subtracted.

\section{Tissue specimens}

Formalin-fixed paraffin-embedded tissues were obtained from the University of Pittsburgh Tumor Bank. We found only two welldefined prostate adenocarcinomas with liver metastases and none from the Cooperative Human Tumor Network, irrespective of other criteria. These studies, blinded for all personal identifiers, were categorised as exemption $4 \mathrm{e}$ by the University of Pittsburgh IRB.

\section{Statistical analysis}

Statistics for all experiments were performed using the Sigma Plot statistical program (Jandel Scientific, Chicago, IL, USA). Independent Student's $t$-test was utilised to determine a statistical difference between experimental and the controls for individual experiments, with significance generally informed at $P<0.05$.

\section{RESULTS}

E-cadherin is generally lost from the primary tumour cells of metastatic carcinomas, with the result that these cells can now detach from the primary tumour mass and attain a nonpolarised, migratory phenotype (Bates and Mercurio, 2005). Unlike many other tumour suppressors, the E-cadherin gene is neither deleted nor mutated, but rather the downregulation appears to result from epigenetic signals, opening the possibility that this loss of expression may be reverted later during the metastatic cascade (Yates et al, 2007). Intriguingly, E-cadherin expression has been noted on metastases of human carcinomas (Rubin et al, 2001). Thus, we proposed that E-cadherin re-expression coincided with distant metastases, enabling the prostate carcinoma cells to interact with the ectopic parenchyma (Yates et al, 2007). We tested this postulate using liver hepatocytes, as liver is the epithelial organ most commonly colonised by metastatic prostate carcinoma (Ewing, 1922; Shah et al, 2004).

We found that co-culturing DU-145 and PC-3 carcinoma cells with rat hepatocytes resulted in increased E-cadherin expression and decreased EGFR expression (Figure 1A). This occurred over a 6-day period in both cell types, with significant differences by day 2 (Figure $1 \mathrm{~A}$ and $\mathrm{B}$ ). The increase in E-cadherin protein levels was reflected by similarly elevated mRNA levels (Figure 1B), though the appearance of mRNA may lag by about 
A

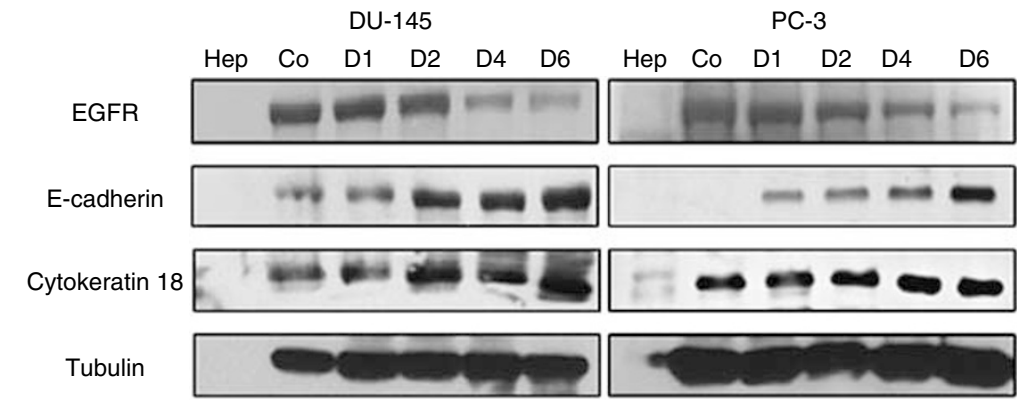

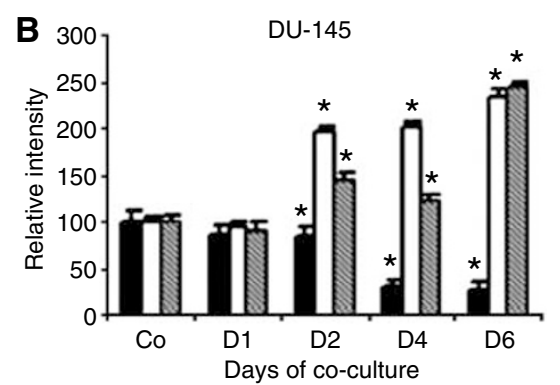

C

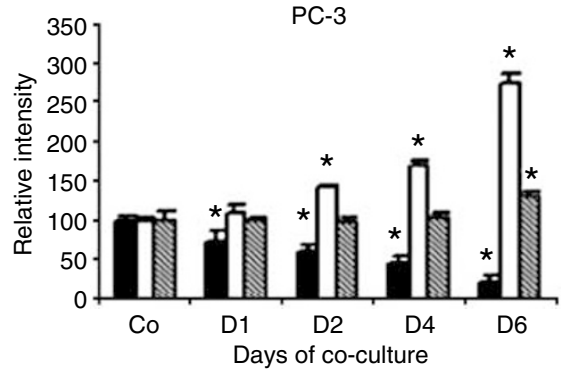

PC-3

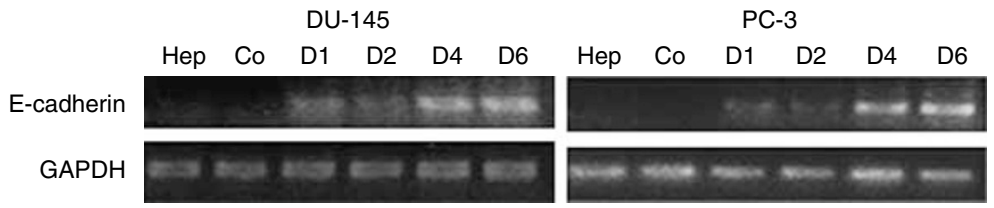

Figure I Co-culture of human prostate cancer cell with rat hepatocytes reversed E-cadherin expression. DU-145 or PC-3 (A) cells were co-cultured in the presence of primary rat hepatocytes over a 6-day period. Hepatocytes and single cultures were lysed before co-cultures. On days I, 2, 4 and 6, coculture lysates were immunoblotted with indicated human selective antibodies: anti-E-cadherin, anti-EGFR antibody, anti-cytokeratin I8 and anti-tubulin (as the loading control). (B) Densitometry of immunoblots in DU-I 45 and PC-3 cells co-cultures ( $\mathbf{a})$ EGFR, ( $\square$ ) E-cadherin () and Cytokeratin I8; shown are the mean \pm s.d. of three blots with day 0 being at $100(* P<0.05$ from C0). Cellular levels of E-cadherin mRNA in DU- 145 or PC-3 (C) cells were analysed by RT-PCR using GAPDH as a loading control. In $\mathbf{A}$ and $\mathbf{B}$, Co (for control) are an equal number of prostate carcinoma cells incubated for I day in the absence of hepatocytes. The Hep (hepatocytes) were an equal number of hepatocytes as for the co-cultures. The first three lanes were all lysed at the same time. Shown are representative of at least three experiments.

1 day. This time differential may imply two levels of control - one at protein degradation and the other at de novo transcriptional potential.

Since loss of E-cadherin and increased EGFR signalling are known markers of invasive mesenchymal cancer cells (Wong and Gumbiner, 2003), we asked if the E-cadherin re-expression and EGFR decreases were accompanied with another known epithelial cell marker. Cytokeratin 18 expression, a marker of mature epithelial cells, increased over the 6-day period in both DU-145 and PC-3 cell lines suggesting a reversion of mesenchymal phenotype characteristic of these cell lines (Figure 1A). This implied a generalised reversion to a more differentiated phenotype in the presence of hepatocytes.

Looking more closely at the subcellular localisation of this Ecadherin and EGFR expression, freshly isolated GFP-expressing primary rat hepatocytes were allowed to adhere $24 \mathrm{~h}$ before seeding of the RFP-expressing prostate cancer cells. As expected, immunofluorescence showed increases in E-cadherin and $\beta$ catenin and inversely related decreases in EGFR expression in the presence of hepatocytes. Unexpectedly however, early cocultures revealed E-cadherin expression at the prostate cancer cell periphery juxtaposed to the hepatocytes during attachment (Figure $2 \mathrm{~A}$ and $\mathrm{B}$ ). $\beta$-Catenin was also localised to the membrane area in these cells further suggesting a functional E-cadherin linkage at the interface of these two cell types. To our knowledge, E-cadherin interaction among different cell types has not been noted previously, thus we consider these interactions as potentially heterotypic in terms of cell types. While various other proteins (e.g. selectins and integrins) have been implicated in carcinoma attachment, heterotypic cell-cell interaction via E-cadherin has not. Therefore, the ability of prostate cancer cells to utilise Ecadherin to bind to hepatocytes was tested by assessing cell-cell adhesion between adherent hepatocytes and prostate carcinoma cells using the centrifugal assay for cell adhesion (McClay et al, 1981). Both DU-145 and PC-3 cells, calcein-AM-labelled, bound to the hepatocytes to a limited but statistically significant degree, and this binding was eliminated by an E-cadherin blocking antibody (Figure 3A-E). As the cell-cell adhesion of the prostate carcinoma cells was limited, though real, we sought to increase the levels of Ecadherin on these cells. For this we used the EGFR kinase inhibitor, PD153035, that we had shown earlier to promote E-cadherin expression in DU-145 cells within 24-48 h (Yates et al, 2005), and confirmed in PC-3 cells at $48 \mathrm{~h}$ (Figure $3 \mathrm{C}$ ). This 48 -h pretreatment with PD153035 increased prostate carcinoma cell binding to the hepatocyte monolayer (Figure $3 \mathrm{~A}-\mathrm{E}$ ).

The above findings implicate EGFR signalling as potentially negatively regulating E-cadherin levels. As this was reminiscent of the situation with LHRH receptor transmodulation of autocrine EGFR signalling in DU-145 cells (Yates et al, 2005), we asked whether a similar situation might be functioning in this setting. We expressed the PKC transmodulation-resistant EGFR A654 variant (Welsh et al, 1991) in the DU-145 cells. Co-culturing these cells with hepatocytes did not lead to decreases in EGFR or increases in E-cadherin and cytokeratin 18 expression (Figure 4). If anything, the DU-145 A654 cells exhibited a decrease in E-cadherin expression over 6-day period, which would be expected since 
A
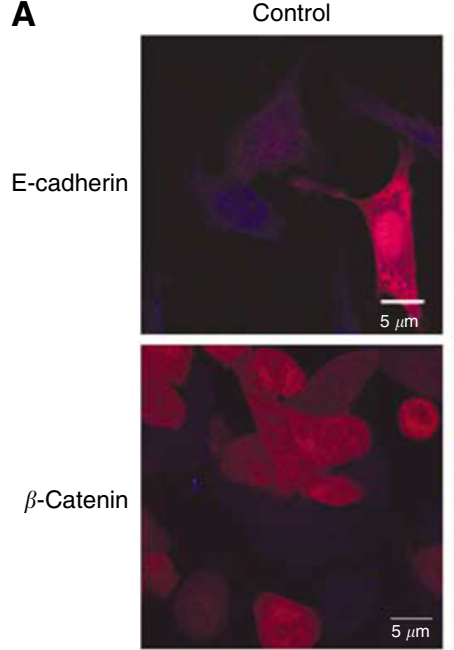

$\overline{5 \mu \mathrm{m}}$

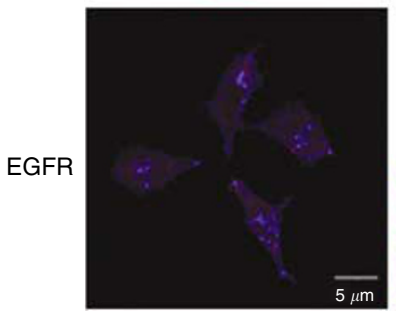

Day 3
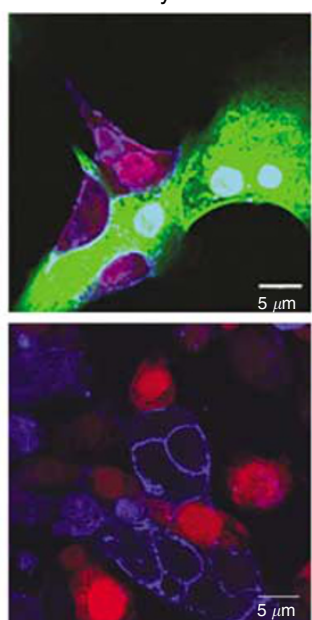

Day 6
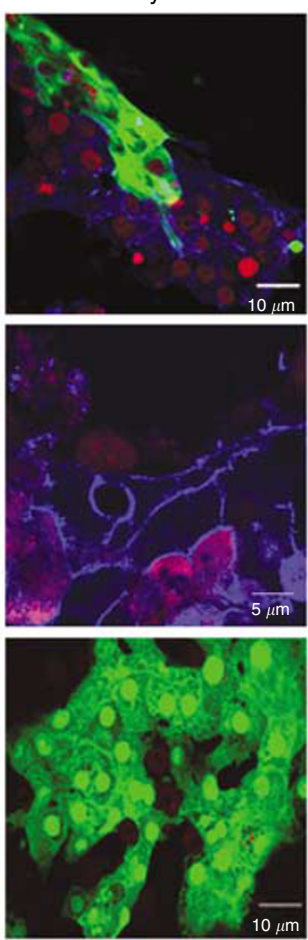

B

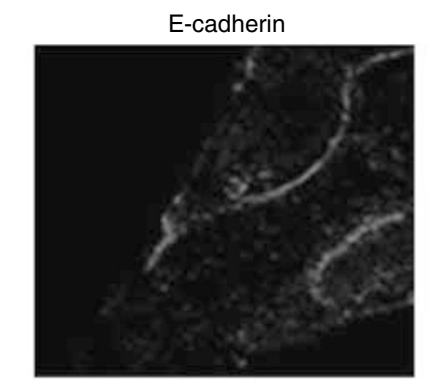

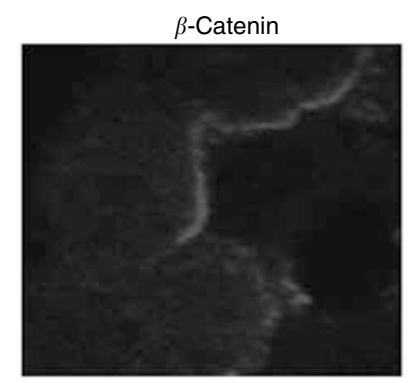

Figure 2 Immunofluorescence of co-cultures show subcellular location of E-cadherin re-expression. (A) Immunofluorescence of DU-I45 RFP (red) and GFP (green) primary rat hepatocytes were stained with human-specific anti-E-cadherin, pan-species anti- $\beta$-catenin or human-specific anti-EGFR antibody. Cy5 secondary antibody (blue) was used for respective primary antibodies. Note the gain of blue (E-cadherin) in the RFP/red prostate cells in the top and middle rows, and the loss of blue (EGFR) in the bottom row. (B) The blue channel only of the lower left inset in the E-cadherin and $\beta$-catenin staining on day 2 is shown in black and white to demonstrate the localisation of the human E-cadherin in the prostate carcinoma cells to the interface with the hepatocytes. In the middle row, the hepatocytes were from WT and not GFP rats, so as not to interfere with the antibody staining as the anti- $\beta$-catenin detected both human and rat. However, the presence of $\beta$-catenin upregulation in the DU-I45 cells is noted by a violet color and the membranous pattern at the hepatocyte-prostate carcinoma cell interface. Shown are representative of at least three experiments.

EGFR signalling continued unabated, and EGFR activity is well documented as leading to E-cadherin downregulation.

These data present a potential metastatic target organ-specific regulation of prostate carcinoma cell phenotype. However, to validate whether this may occur in de novo human tumours, we obtained human liver tissue from two patients with prostate cancer metastases to the liver and examined the expression of E-cadherin in these tumours by immunohistochemistry. E-cadherin staining was significant in the tumour nodules within the liver (Figure 5A). This increased expression was accompanied by increases in Ecadherin-associated adhesion molecules $\alpha, \beta$ and p120 catenin as well (Figure 5A). Central to our model of inverse relationship between E-cadherin expression and EGFR (Yates et al, 2005), we were able to observe a lack of total and active EGFR expression in these tumours (Figure 6A). To molecularly determine if these tumours reverted to a more epithelial phenotype, we stained for the epithelial marker cytokeratin 18 and the mesenchymal marker vimentin. Akin to our in vitro findings, these tumours largely express cytokeratin and lack vimentin expression (Figure 6B). In the absence of the primary tumours, from which these metastases derived, we cannot state that this represents a reversion in the phenotypic profile, but given the widespread finding of EMT in invasive and metastatic primary tumour, it does suggest that there may be cancer cell phenotypic variability as result of the metastatic microenvironment.

\section{DISCUSSION}

Loss of E-cadherin is so widely observed in advanced carcinomas that the E-cadherin molecule is considered a tumour suppressor (Wong and Gumbiner, 2003). This conception of E-cadherin is supported by forced re-expression of E-cadherin resulting in diminished tumourigenic potential in experimental tumour systems (Jawhari et al, 1999; Yates et al, 2005). However, unlike classical tumour suppressors (e.g. p53 and Rb), E-cadherin loss occurs by epigenetic downregulation or transcriptional silencing, rather than genetic deletion or mutation (Graff et al, 1995). As the 
A

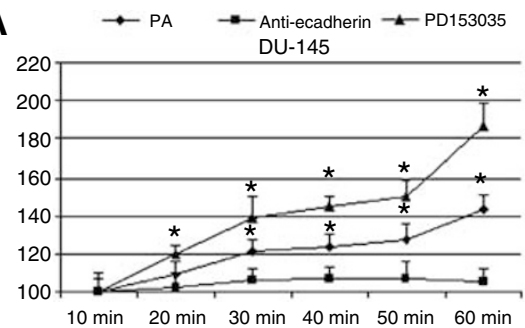

B
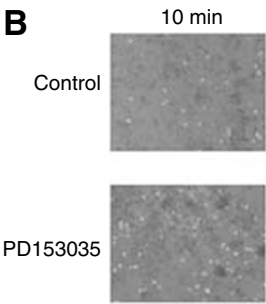

$30 \mathrm{~min}$
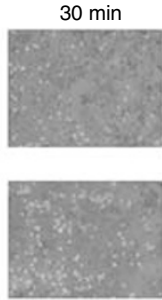

C

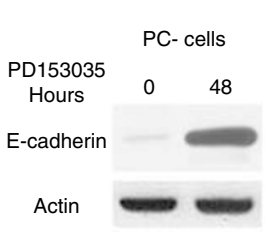

D $\rightarrow$ Control $¥$ Anti-ecadherin $*$ PD153035

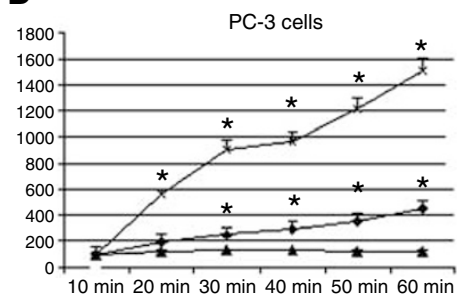

$60 \mathrm{~min}$

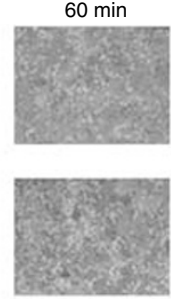

E

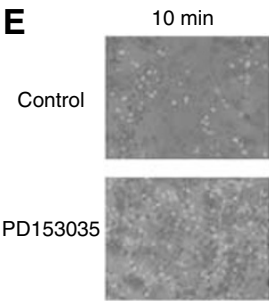

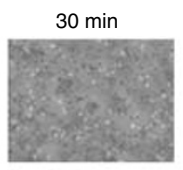

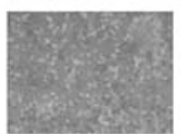

$60 \mathrm{~min}$

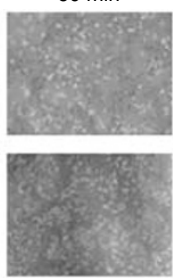

Figure 3 Human prostate cancer cells form E-cadherin-mediated heterotypic interactions with hepatocytes. DU-I 45 (A and B) cells or PC-3 (C-E) cells fluorescently labelled with Calcein A were incubated for $48 \mathrm{~h}$ with PDI53035 or E-cadherin blocking antibody and seeded onto a monolayer of hepatocytes from non-GFP-expressing rats to analyse their ability to adhere. Cell binding was assessed by fluorescent intensity using a plate fluorometer and visually verified under a fluorescent light microscope. Y-axis is arbitrary fluorescent units. Data represent mean of three experiments performed in triplicate; s.e. $* P<0.05$. Shown are sample representative fields to show the bound tumour cells (converted to white dots) overlying the hepatocytes in B and $\mathbf{E}$. (C) PC3 cells were exposed to PDI 53035 for $48 \mathrm{~h}$ with a resultant upregulation of E-cadherin as shown by immunoblotting. This is similar to our previously published finding with DUI45 cells (Yates et al, 2005).

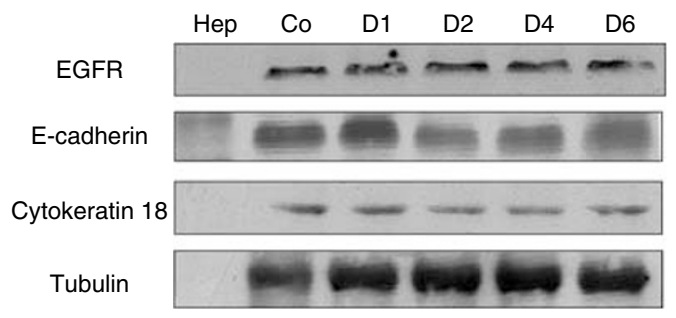

Figure 4 DU-I45 cells expressing a PKC transattenuation-resistant EGFR (A654) are resistant to hepatocyte-induced E-cadherin re-expression. DU-I 45 A654 cells co-culture lysates were immunoblotted with an antibody selective for human E-cadherin, EGFR, cytokeratin 18 or tubulin. The legend is as with Figure I, Co (control) DUI 45 A654 cells and Hep (hepatocytes) only. Shown is one of two representative blot series.

mechanisms which diminish E-cadherin are reversible, this opens the question as to whether there are situations in which reexpression of E-cadherin and subsequent cell-cell adhesion may promote tumour progression. Herein, we provide proof of principle that parenchymal cells from a tissue frequently targeted for prostate carcinoma metastasis, the liver (Ewing, 1922; Lind et al, 2004; Shah et al, 2004), signal this re-expression of Ecadherin.

Downregulation of E-cadherin occurs frequently in the primary prostate tumour coincident with aggression and spread (Gingrich et al, 1996; Kallakury et al, 2001). However, expression in distant metastases has been noted in some prostate cancer metastases (Rubin et al, 2001) but not in other studies in metastases to the bone (Bryden et al, 2002). Our findings herein, appear consistent with the re-expression, though the liver environment is likely substantially different from that of the bone in terms of signals to the hepatocytes and requirements by the metastatic tumour cells. It must also be noted that there is precedence for E-cadherin expression on disseminated carcinomas, as ovarian carcinoma dissemination throughout the peritoneal cavity shows increased levels of E-cadherin and its attendant catenins (Imai et al, 2004). Again, though, the applicability to metastatic spread of an organconfined prostate cancer to a distant-defined organ compartment may not be comparable to the spread of ovarian carcinoma throughout an adjacent open cavity.

The findings herein raise two questions that need to be explored in depth in future investigations. First and most obvious, the mechanism by which the hepatocytes signal for E-cadherin reexpression needs to be defined. Our initial findings strongly suggest a link to downregulation of autocrine EGFR signalling. EGFR levels change inversely to the E-cadherin levels, and direct inhibition of EGFR signalling leads to increased E-cadherin expression (Yates et al, 2005). More directly, expression of an EGFR variant that is resistant to PKC-mediated attenuation renders the DU-145 cells impervious to hepatocyte-induced Ecadherin expression. This presages the ultimate issues of the nature of the signals from the hepatocytes and which carcinoma intracellular processes actuate the E-cadherin re-emergence. The intracellular events may be complex and involve both loss of downregulation at the protein level and reversion of transcriptional suppression. That EGFR activity is known to disrupt the E-cadherin/catenin complex leading to E-cadherin protein destabilisation and degradation (Ackland et al, 2003) and the impression that E-cadherin protein expression may precede increased mRNA suggest that protein downregulation is part of the answer. Although the intracellular mechanisms of E-cadherin re-expression are undoubtedly complex, these findings are novel and provide insight of prostate cancer cell behavior within a target soft organ.

One caveat that deserves specific mention is that EGFR signalling contributes to numerous aspects of prostate tumour progression. For instance, autocrine EGFR-mediated motility promotes tumour invasion and metastasis and tumour cell proliferation and survival (Wells, 2000). Thus, targeting EGFR kinase may have effects other than promoting the metastatic engraftment proposed herein by the upregulation of E-cadherin. For instance, the recent report of EGFR kinase inhibitors limiting prostate tumour dissemination in experimental models (Angelucci et al, 2006) is likely due to inhibiting pathways and cell behaviors before the proposed upregulation of E-cadherin at the metastatic site, though re-expression of E-cadherin at the primary tumour site would also limit spread by preventing initial detachment (Jawhari et al, 1999; Lowy et al, 2002; Wong and Gumbiner, 2003). 

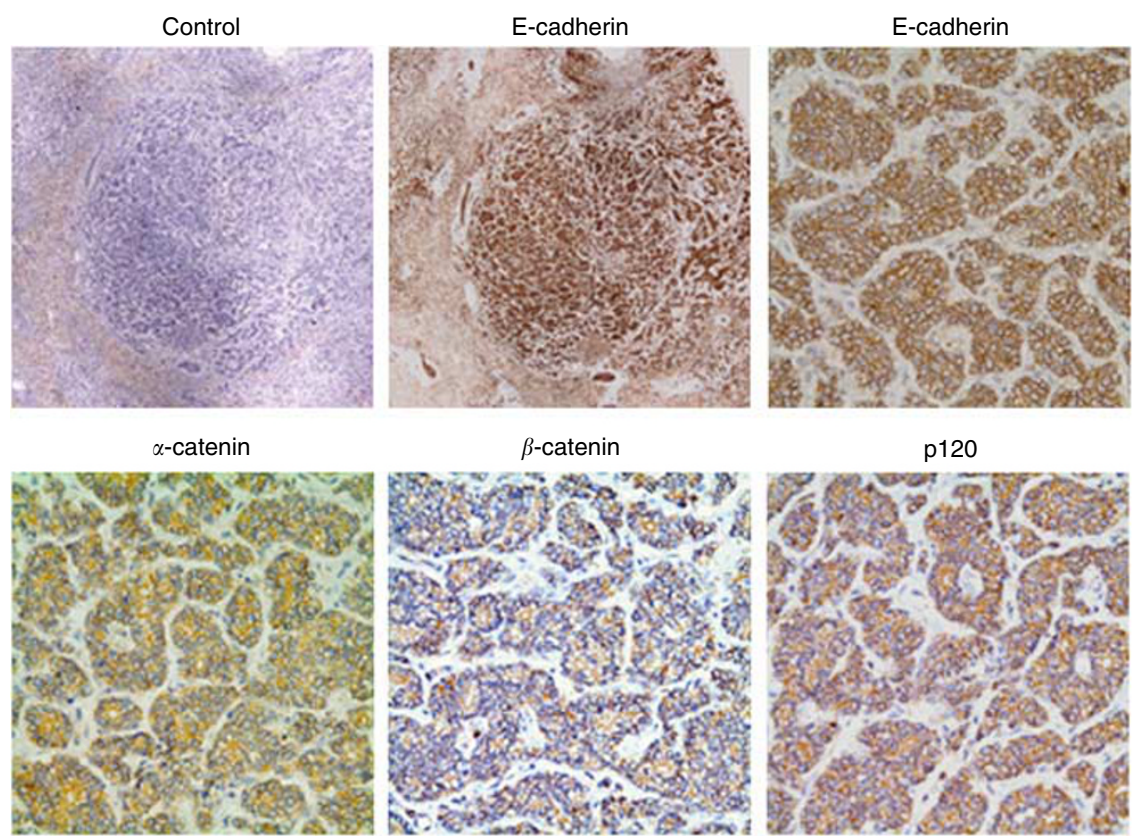

Figure 5 Human prostate cancer metastases to liver show expression of cell-cell adhesion molecules. Formalin-fixed, paraffin-embedded tissues were obtained from two well-defined prostate adenocarcinomas with liver metastasis. Tissues were stained with indicated antibodies, Secondary antibody, antimouse only as the staining control (top left; $3700 \mu \mathrm{m}^{2}$ ), anti-E-cadherin (top centre; $3700 \mu \mathrm{m}^{2}$ and top right; $300 \mu \mathrm{m}^{2}$ ), anti- $\alpha$-catenin (bottom right; $300 \mu \mathrm{m}^{2}$ ), anti- $\beta$-catenin (bottom centre; $300 \mu \mathrm{m}^{2}$ ) and anti-p 120 (bottom right; $300 \mu \mathrm{m}^{2}$ ). Shown are representative of repeated stainings; the other metastasis presented similar findings.
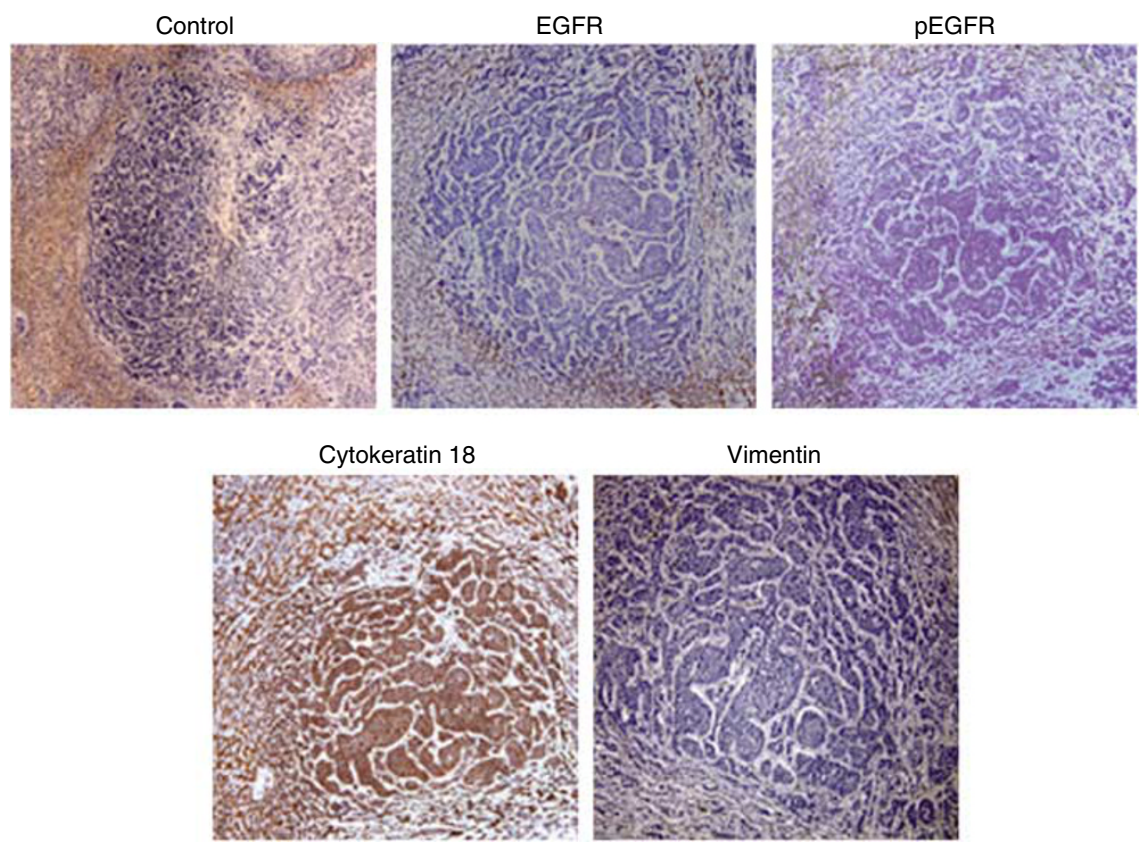

Figure 6 Human prostate cancer metastases show reversion of metastatic markers. Tissues were stained with anti-rabbit (top left; I $400 \mu \mathrm{m}^{2}$ ) anti-EGFR (top centre; I $400 \mu \mathrm{m}^{2}$ ), anti-phosphotyrosyl-EGFR (activated EGFR) (top right; I $400 \mu \mathrm{m}^{2}$ ), anti-vimentin (bottom left; I $400 \mu \mathrm{m}^{2}$ ) and anti-cytokeratin I8 (bottom left; $1400 \mu \mathrm{m}^{2}$ ). Shown are representative of repeated stainings; the other metastasis presented similar findings.

The second question of what selective advantage is conferred by E-cadherin re-expression remains in the speculative realm. Our finding and those reported in the literature (Rubin et al, 2001; Kowalski et al, 2003) or carcinoma metastases presenting Ecadherin and differentiation-related cytokeratins, along with low to absent levels of vimentin and EGFR (total and active), cannot discern whether these metastases arose from a subset of primary tumour cells that never underwent a phenotypic shift or from the majority of aggressive cells that underwent the so-called epithelial-mesenchymal transition. However, the fact that experimental prostate cancer cell lines, DU-145 and PC-3, exhibited similar phenotypic shifts when cultured with hepatocytes implies a survival or growth advantage in these ectopic sites that is at odds with that seen for the EMT tumour cells in the primary locale. Recently, this has been supported experimentally by the finding that epithelial variants of a bladder carcinoma cell line presented 
greater seeding of metastatic sites when introduced into the circulation than the mesenchymal parental line, whereas the parental mesenchymal tumour line possessed increased metastatic capabilities from an orthotopic location (Chaffer et al, 2006). One can speculate that in a metastatic tumour microenvironment lacking many of the prostate-specific survival signals, E-cadherinmediated linkages provide critical survival signals, by linking to both other carcinoma cells and even the hepatocytes themselves. Such studies, which lie beyond the realm of the current communication, are underway.

In conclusion, we propose a model in which the differentiation phenotype of metastatic prostate carcinoma cells is plastic in response to the microenvironment. In the primary tumour setting, E-cadherin expression is downregulated as part of the EMT that allows disaggregation from the primary mass enabling invasive migration and distant dissemination. However, once the tumour cells reach the ectopic microenvironment, numerous signals critical for survival and/or growth are absent. The metastatically competent subset of tumour cells interpret initiating signals from the target organ, in this case the liver, to re-express a more differentiated phenotype, or to undergo a mesenchymalepithelial reverse transition. This redifferentiation could provide for both homotypic and heterotypic cell-cell adhesion with concomitant survival signals. While such a redifferentiation would be expected to limit tumour cell proliferation and local invasiveness, it may be key to prevent tumour cell apoptosis in the absence of a supportive orthotopic microenvironment. In-depth exploration is necessary not only to determine if such metastatic carcinoma redifferentiation occurs in de novo metastasis of human tumours, but also to explain the relative resistance to chemotherapy of metastases and even the concept of tumour dormancy.

\section{ACKNOWLEDGEMENTS}

We thank Linda Griffith and George Michalopoulos for discussions and suggestions. These studies were supported by grants from the VA Merit Award Program and the DoD Congressionally Directed Medical Research Program.

\section{REFERENCES}

Ackland ML, Newgreen DF, Fridman M, Waltham MC, Arvanitis A, Minichiello J, Price JT, Thompson EW (2003) Epidermal growth factorinduced epithelio-mesenchymal transition in human breast carcinoma cells. Lab Invest 83: $435-448$

Angelucci A, Gravina GL, Rucci N, Millimaggi D, Festuccia C, Muzi P, Teti A, Vicentini C, Bologna M (2006) Suppression of EGF-R signaling reduces the incidence of prostate cancer metastasis in nude mice. Endocr Relat Cancer 13: $197-210$

Bates RC, Mercurio AM (2005) The epithelial-mesenchymal transition (EMT) and colorectal cancer progression. Cancer Biol Ther 4: 365-370

Brabletz T, Jung A, Reu S, Porzner M, Hlubek F, Kunz-Schughart LA, Knuechel R, Kirchner T (2001) Variable beta-catenin expression in colorectal cancers indicates tumor progression driven by the tumor environment. Proc Natl Acad Sci USA 98: 10356-10361

Bryden AA, Hoyland JA, Freemont AJ, Clarke NW, Schembri-Wismayer D, George NJ (2002) E-cadherin and beta catenin are down-regulated in prostatic bone mestatases. BJU Intl 89: $400-403$

Chaffer CL, Brennan JP, Slavin JL, Blick T, Thompson EW, Williams ED (2006) Mesenchymal-to-epithelial transition facilitates bladder cancer metastasis: role of fibroblast growth factor receptor-2. Cancer Res 66: $11271-11278$

Condeelis J, Segall JE (2003) Intravital imaging of cell movement in tumours. Nat Rev Cancer 3: 921-930

Davies G, Jiang G, Mason MD (2000) Cell-cell adhesion molecules and signaling intermediates and their role in the invasive potential of prostate cancer cells. J Urol 163: 985-992

Ewing J (1922) Tumors of prostate. In Neoplastic Diseases pp 784-785. Philadelphia and London: WB Saunders Company

Gingrich JR, Barrios RJ, Morton RA, Boyce BF, DeMayo FJ, Finegold MJ, Angelopoulou R, Rosen JM, Greenburg NM (1996) Metastatic prostate cancer in a transgenic mouse. Cancer Res 56: 4096-4102

Graff JR, Herman JG, Lapidus RG, Chopra H, Xu R, Jarrard DF, Isaacs WB, Pitha PM, Davidson NE, Baylin SB (1995) E-cadherin expression is silenced by DNA hypermethylation in human breast and prostate carcinomas. Cancer Res 55: 5195-5199

Hurtubise A, Momparler RL (2004) Evaluation of antineoplastic action of 5-aza-2'-deoxycytidine (Dacogen) and docetaxel (Taxotere) on human breast, lung and prostate carcinoma cell lines. Anticancer Drugs 15: $161-167$

Imai T, Horiuchi A, Shiozawa T, Osada R, Kikuchi N, Ohira S, Oka $\mathrm{K}$, Konishi I (2004) Elevated expression of E-cadherin and alpha-, beta-, and gamma-catenins in metastatic lesions compared with primary epithelial ovarian carcinomas. Human Pathol 33: 1469-1476

Jawhari AU, Farthing MJ, Pignatelli M (1999) The E-cadherin/epidermal growth factor receptor interaction: a hypothesis of reciprocal and reversible control of intercellular adhesion and cell proliferation. J Pathol 187: $155-157$
Kallakury BV, Sheehan CE, Winn-Deen E, Oliver J, Fisher HA, Kaufman RP, Ross JS (2001) Decreased expression of catenins (alpha and beta), p120 CTN, and E-cadherin cell adhesion proteins and E-cadherin gene promoter methylation in prostatic adenocarcinomas. Cancer 92: $2786-2795$

Kowalski PJ, Rubin MA, Kleer CG (2003) E-cadherin expression in primary carcinomas of the breast and its distant metastases. Breast Cancer Res 5 : $\mathrm{R} 217$ - R222

Lind GE, Thorstensen L, Lovig T, Meling GI, Hamelin R, Rognum TO, Esteller M, Lothe RA (2004) A CpG island hypermethylation profile of primary colorectal carcinomas and colon cancer cell lines. Mol Cancer 3: 28

Lowy AM, Knight J, Groden J (2002) Restoration of E-cadherin/betacatenin expression in pancreatic cancer cells inhibits growth by induction of apoptosis. Surgery 132: $141-148$

McClay DR, Wessel GM, Marchase RB (1981) Intercellular recognition: quantitation of initial binding events. Proc Natl Acad Sci USA 78: 4975 4979

Rubin MA, Mucci NR, Figurski J, Fecko A, Pienta KJ, Day ML (2001) E-cadherin expression in prostate cancer: a broad survey using highdensity tissue microarray technology. Hum Pathol 32: 690-697

Seglen PO (1976) Preparation of isolated rat liver cells. Methods Cell Biol 13: $29-83$

Shah RB, Mehra R, Chinnaiyan AM, Shen R, Ghosh D, Zhou M, Macvicar GR, Varambally S, Harwood J, Bismar TA, Kim R, Rubin MA, Pienta K] (2004) Androgen-independent prostate cancer is a heterogeneous group of diseases: lessons from a rapid autopsy program. Cancer Res 64: 92099216

Thiery JP (2002) Epithelial-mesenchymal transitions in tumour progression. Nat Rev Cancer 2: 442-454

Wells A (2000) Tumor invasion: role of growth factor-induced cell motility. Adv Cancer Res 78: $31-101$

Welsh JB, Gill GN, Rosenfeld MG, Wells A (1991) A negative feedback loop attenuates EGF-induced morphological changes. J Cell Biol 114: 533-543

Wheeler JM (2005) Epigenetics, mismatch repair genes and colorectal cancer. Ann R Coll Surg Engl 87: 15-20

Wong AS, Gumbiner BM (2003) Adhesion-independent mechanism for suppression of tumor cell invasion by E-cadherin. J Cell Biol 161: $1191-1203$

Yates C, Shepard C, Papworth G, Dash A, Beer-Stolz D, Griffith L, Wells A (2007) Novel three-dimensional organotypic liver bioreactor to directly visualize early events in metastatic progression. Adv Cancer Res 96 (in press)

Yates C, Wells A, Turner T (2005) Luteinising hormone-releasing hormone analogue reverses the cell adhesion profile of EGFR overexpressing DU-145 human prostate carcinoma subline. Br J Cancer 92: $366-375$ 